\title{
ON THE LATTICE THEORY OF FUNCTION SEMI-NORMS
}

\author{
I.E. SCHOCHETMAN \\ and \\ S.K. TSUI \\ Department of Mathematical Sciences \\ Oakland University \\ Rochester, Michigan \\ 48063 \\ (Received October 11,1982 )
}

ABSTRACT. We consider the lattice of function semi-norms over a measure space, as well as certain distinguished subsets. We determine which subsets are sublattices and, in turn, which of these are Dedekind complete. We also investigate the extent to which the distributive and DeMorgan Laws are valid in this setting. KEY WORDS AND PHRASES. Function semi-norm, associate semi-norm, infimum, supremum, Riesz-Fisher property, strong and weak Fatou properties, infinite triangle inequality, absolutely continuous norm, (sub-) lattice, Dedekind completeness, distributive laws, DeMorgan's Laws.

1980 AMS MATHEMATICS SUBJECT CLASSIFICATION CODES. Primary: 06A23, 06A35, 46E30 Secondary: 06A40, 46B05

\section{INTRODUCTION.}

In this paper, we investigate the sublattice structure of the lattice of semi-norms on a fixed measure space.

Section 2 is devoted to establishing the necessary preliminaries. In Section 3, we distinguish the semi-norms of interest, namely those having either the Riesz-Fisher, weak or strong Fatou properties, those satisfying the infinite triangle inequality and those which are of absolutely continuous norm. In determining the sublattice status of each of these collections, we are also interested in Dedekind completeness for each of these, as well as for the space of all semi-norms. In particular, when a subset is closed under arbitrary suprema or infima, we wish to know the specific description of these extrema. The answers to these questions in the case of the supremum are essentially known. 
For the sake of completeness, these are summarized in Section 4. However, the answers to these questions in the case of the infimum are hardly known at all. In fact, the notion of infimum is not unique; it depends on which sublattice is being considered. The main difficulty here involves the infimum of an arbitrary collection of function semi-norms. There are three candidates for this notion which we define and study in Section 5. Here we also determine which infimum goes with which sublattice. This is the main section of this paper. In Section 6, we are then able to determine (1) whether a given subset of semi-norms is a sublattice and (2) if it is complete.

In Section 7, we turn our attention to the DeMorgan Laws. As we shall see, the validity of these laws depends on which of the two laws is being considered--as we11 as its interpretation.

In concluding, Section 8 observes that the sublattices under consideration are not distributive in general and also shows that there is a connection between the lattice theory of function semi-norms and finite sums of such semi-norms.

\section{PRELIMINARIES.}

Here we recall the preliminary results we will require throughout the remainder of this paper. A general reference for this section is [5].

Let $(x, S, \mu)$ be a fixed sigma-finite measure space and $[0, \infty]$ the extended, non-negative real numbers. As usual, the real numbers and the natural numbers will be denoted by $\mathbb{R}$ and $\mathbb{N}$, equipped with their usual measure structures. If $C$ is the complex numbers, define

$$
M=\{f: X \rightarrow C: f \text { is } \mu \text {-measurable }\}
$$

and

$$
\mathrm{M}^{+}=\{\mathrm{f}: \mathrm{X} \rightarrow[0, \infty]: \mathrm{f} \text { is } \mu \text {-measurable }\} \text {. }
$$

In general, we will not distinguish between elements of $M$ or $\mathrm{M}^{+}$which are equal almost everywhere.

In will also be convenient to denote $x \in X: f(x) \neq 0$ by $\operatorname{supp}(f)$. DEFINITION 2.1. A(function) semi-norm on $M^{+}$is a mapping $\rho: M^{+} \rightarrow[0, \infty]$ satisfying : 
(i) $\rho(0)=0$.

(ii) $\rho(a f)=a \rho(f), a \geq 0, f \varepsilon M^{+}$.

(iii) $\rho(f+g) \leq \rho(f)+\rho(g), f, g \in M^{+}$.

(iv) $\rho(f) \leq \rho(g)$, whenever $f \leq g$, $f, g \in M^{+}$.

The semi-norm $\rho$ is a norm if $\rho(f)=0$ implies $f=0$. Let $P$ denote the set of all semi-norms and $P_{0}$ the subset of all norms (never empty). Of course, $\rho=\|\cdot\|_{p}$ is a norm for each $1 \leq p \leq \infty$.

Observe that $P$ is canonically partially ordered by

$$
\rho_{1} \geq \rho_{2} \quad \text { if } \quad \rho_{1}(f) \geq \rho_{2}(f) \text {, f } \varepsilon M^{+} \text {. }
$$

EXAMPLE 2.2. For $f$ in $M^{+}$, define $\theta(f)=0$. Then $\theta \varepsilon P-P_{0}$. Also, for $f$ in $M^{+}$, define $\omega(f)=\infty, f \neq 0, \omega(0)=0$. Then $\omega \varepsilon P_{0}$. Clearly, $\theta$ and $\omega$ are the smallest and largest elements of $P$.

LEMMA 2.3. Let $\rho \in P$ and $g \in M^{+}$. Define $\rho_{g}(f)=\rho(f g), f \in M^{+}$. Then $\rho_{g}$ is a semi-norm. Of particular interest are the cases where

(i) $g$ is the characteristic function $X_{E}$ of a measurable set $E$ or

(ii) $\rho$ is the $\mathrm{L}^{1}$-norm $\|\cdot\|_{1}$.

Now define

$$
L^{\rho}=\{f \& M: \rho(|f|)<\infty\}
$$

and

$$
\mathrm{N}^{\rho}=\{\mathrm{f} \varepsilon \mathrm{M}: \rho(|\mathrm{f}|)=0\}
$$

Then $L^{\rho}$ is a semi-normed linear space with $\mathbf{N}^{\rho}$ a closed subspace. Thus, $L^{\rho}=L^{\rho} / \mathrm{N}^{\rho}$ is a normed linear space. If $\rho=\|\cdot\|_{p}$, then $L^{\rho}$ is the familiar $\mathrm{L}^{\mathrm{P}}$-space.

EXAMPLE 2.4. Let $g=\chi_{E}$ as in 2.3, with $\rho \varepsilon P$. Then $\rho_{E}=\rho_{g} \leq \rho$, so that $L^{\rho} \subseteq L^{\rho}$. On the other hand, for $E=X$, we have $L^{\rho}=L^{\rho_{E}}$. Hence, in general,

$$
L^{\rho}=\cap\left\{L^{\rho}: E \varepsilon S\right\}
$$


REMARK 2.5. If $\rho_{2}, \rho_{2} \varepsilon P$, then the pointwise $\operatorname{supremum} \sup \left(\rho_{1}, \rho_{2}\right)$ of $\rho_{1}, \rho_{2}$ belongs to $P[5, p .442]$. This is not the case for the pointwise infimum. DEFINITION 2.6. Let $\rho_{1}, \rho_{L} \varepsilon \mathrm{P}$. For $\mathrm{f}$ in $\mathrm{M}^{+}$, define

$$
\inf \left(\rho_{1}, \rho_{2}\right)(f)=\inf \left\{\rho_{1}\left(f_{1}\right)+\rho_{2}\left(f_{2}\right): f_{1}, f_{2} \varepsilon M^{+}, f_{1}+f_{2}=f\right\} .
$$

The function $\inf \left(\rho_{1}, \rho_{2}\right)$ is a semi-norm and is the infimum of $\rho_{1}, \rho_{2}$ in $P$ $[5, \mathrm{Ex} .71]$

In what follows, it will be convenient to write $\rho_{1} \vee \rho_{2}$ for $\sup \left(\rho_{1}, \rho_{2}\right)$ and $\rho_{1} \wedge \rho_{2}$ for $\inf \left(\rho_{1}, \rho_{2}\right)$. Now let

$$
F=\left\{\rho: M^{+} \rightarrow[0, \infty]: \rho(0)=0\right\}
$$

(Note that an element of $F$ need not be a semi-norm.) For such $\rho$, we may define the associate $\rho^{\prime}$ by

$$
\rho^{\prime}(f)=\sup \left\{\int_{X}(f g) d \mu ; \rho(g) \leq 1\right\}, \text { f } \varepsilon M^{+} \text {. }
$$

Observe that the supremum is taken over a non-empty set. The function $\rho^{\prime}$ is a semi-norm, i.e., $\rho^{\prime} \varepsilon$ P. In particular, $\theta^{\prime}=\omega, \omega^{\prime}=\theta$ and $\left(\rho_{p}\right)^{\prime}=\rho_{p^{\prime}}$, where $1 / p+1 / p^{\prime}=1,1 \leq p \leq \infty$.

LEMMA 2.7. (i) If $\rho_{1}, \rho_{2} \varepsilon P$ and $\rho_{1} \leq \rho_{2}$, then $\rho_{1}^{\prime} \geq \rho_{2}^{\prime}$. (ii) If $\rho \varepsilon P$, then $\rho^{\prime \prime} \leq \rho$ and $p^{(n)}=p^{(n+2)}, n=1,2,3, \ldots$.

LEMMA 2.8. (Finite DeMorgan Laws) If $\rho_{1}, \rho_{2} \varepsilon \mathrm{P}$, then $\left(\rho_{1} \wedge \rho_{2}\right)^{\prime}=\rho_{1}^{\prime} \vee \rho_{2}^{\prime}$ and $\left(\rho_{1} \vee \rho_{2}\right)^{\prime}=\rho_{1}^{\prime} \wedge \rho_{2}^{\prime} \cdot$

PROOF. The first law is we11-known [5,Ex.71.2]. The second law is much less well-known; in fact, there appears to be no proof of it in the literature. The proof we give next is due to Luxemburg [3]. We are grateful to Professor Luxemburg for permitting its inclusion here.

For $\rho_{1}, \rho_{2} \in P$, we have $\rho_{1}, \rho_{2}<\rho_{1} \vee \rho_{2}$, so that $\rho_{1}^{\prime} \rho_{2}^{\prime} \geq\left(\rho_{1} \vee \rho_{2}\right)^{\prime}(1.14)$, i.e., $\left(\rho_{1}^{\prime} \wedge \rho_{2}^{\prime}\right) \geq\left(\rho_{1} \vee \rho_{2}\right)^{\prime}$. To prove the other inequality, let $\rho=\rho_{1} \vee \rho_{2}$ and suppose $\mathrm{f}$ is an element of $M^{+}$such that $\rho^{\prime}(f)<\infty \quad$ (otherwise, equality is trivial). Since $f \in L^{\rho^{\prime}}$, it corresponds to a bounded linear functional 
$\varphi$ on $L^{\rho}$ given by

$$
\varphi(g)=\int_{X} \mathrm{fgd} \mu, g \varepsilon \mathrm{L}^{\rho}
$$

Also, the Banach dual norm $\rho^{*}$ of $\rho$ is equal to $\rho^{\prime}$ on $L^{\rho^{\prime}}$. It then follows from [2, p. 247] that there exist non-negative linear functionals $\varphi_{1}, \varphi_{2}$ on $L^{\rho_{1}}$, $L^{\rho_{2}}$ such that $\varphi=\varphi_{1}+\varphi_{2}$ on $L^{\rho}$ and

$$
\rho^{\prime}(f)=\rho^{*}(f)=\rho^{*}(\varphi)=\rho_{1}^{*}\left(\varphi_{1}\right)+\rho_{2}^{*}\left(\varphi_{2}\right) \text {. }
$$

Since $\varphi_{1}, \varphi_{2} \leq \varphi$ on $L^{\rho}$, it follows from the Radon-Nikodym Theorem that $\varphi_{1}, \varphi_{2}$ are integrals, i.e., there exist functions $\mathrm{f}_{1}, \mathrm{f}_{2} \geq 0$ in $\rho_{1}^{\prime} \rho_{2}^{\rho_{2}^{\prime}}$ respectively such that

$$
\varphi_{i}(g)=\int_{X} g f_{i} d \mu, g \varepsilon L^{\rho} .
$$

Thus, $f_{i} \varepsilon L^{\rho}$ and $\rho_{i}^{*}\left(\varphi_{i}\right)=\rho_{i}^{\prime}\left(f_{i}\right), i=1,2$. Since $\varphi=\varphi_{1}+\varphi_{2}$, it follows that $f=f_{1}+f_{2}$; also, $\rho^{\prime}(f)=\rho_{1}^{\prime}\left(f_{1}\right)+\rho_{2}^{\prime}\left(f_{2}\right)$. Consequent $1 y$, $\rho^{\prime}(f) \geq\left(\rho_{1}^{\prime} \wedge \rho_{2}^{\prime}\right)(f)$, i.e., $\left(\rho_{1} \vee \rho_{2}\right)^{\prime} \geq \rho_{1}^{\prime} \wedge \rho_{2}^{\prime}$.

\section{THE SUBSETS.}

In addition to the subset $P$ of $F$, we will be interested in studying a chain of subsets of $\mathrm{P}$ having certain desirable properties. In this section we recall these properties, show their relationships to each other and consider some general examples. References for this section are $[4,5]$. With the exception of the subset satisfying the infinite triangle inequality, these subsets have been studied before.

DEFINITION 3.1. Let $\rho \varepsilon \mathrm{P}$. Then:

(i) $\rho$ has the Riesz-Fisher Property (RFP) if for each sequence $\left\{f_{n}\right\}$ in $M^{+}$ such that $\Sigma \rho\left(f_{n}\right)<\infty$, it follows that there exists a sequence $\left\{g_{n}\right\}$ in $M^{+}$ such that $\rho\left(\mathrm{f}_{\mathrm{n}}-\mathrm{g}_{\mathrm{n}}\right)=0$ and $\rho\left(\Sigma \mathrm{g}_{\mathrm{n}}\right)<\infty$.

(ii) $\rho$ satisfies the Infinite Triangle Inequality (ITI) if for each sequence $\left\{f_{n}\right\}$ in $M^{+}$it follows that $\rho\left(\Sigma f_{n}\right) \leq \Sigma \rho\left(f_{n}\right)$.

(iii) $\rho$ has the Weak Fatou Property (WFP) if for each sequence $\left\{f_{n}\right\}$ in $M^{+}$ 
such that $\mathrm{f}_{\mathrm{n}} \uparrow \mathrm{f} \varepsilon \mathrm{M}^{+}$, and $\lim \rho\left(\mathrm{f}_{\mathrm{n}}\right)<\infty$, it follows that $\rho(\mathrm{f})<\infty$. (iv) $\rho$ has the Strong Fatou Property (SFP) if for each sequence $\left\{\mathrm{f}_{\mathrm{n}}\right\}$ in $\mathrm{M}^{+}$ such that $\mathrm{f}_{\mathrm{n}} \uparrow \mathrm{f}$, $\mathrm{f} \varepsilon \mathrm{M}^{+}$, it follows that $\lim \rho\left(\mathrm{f}_{\mathrm{n}}\right)=\rho(\mathrm{f})$.

(v) $\rho$ is of Absolutely Continuous Norm (ACN) if $L_{a}^{\rho}=L^{\rho}$, where $L_{a}$ is defined to be the subspace of $f$ in $L^{\rho}$ having absolutely continuous norm. Recall that $\mathrm{f}$ in $\mathrm{L}^{\rho}$ has this property if for each sequence $\left\{\mathrm{f}_{\mathrm{n}}\right\}$ in $\mathrm{M}^{+}$such that $|\mathrm{f}| \geq \mathrm{f}_{\mathrm{n}} \downarrow 0$, the sequence $\left\{\mathrm{p}\left(\mathrm{f}_{\mathrm{n}}\right)\right\}$ converges to 0 .

Let $R$ (resp. $I, W, S$ ) denote the subset of $P$ consisting of the RFP (resp. ITI, WFP, SFP) semi-norms. Also, let A denote the subset of $S$ (equivalently $W$ ) consisting of the ACN semi-norms. Finally, if $B$ is any subset of $P$, let $B_{0}$ denote the set $B \cap P_{0}$ of norms in $B$. Thus, we obtain the subsets $R_{0}, I_{0}, W_{0}, S_{0}, A_{0}$ of $P_{0}$, where $I_{0}=R_{0}[4, T h m .4 .2]$. We then have the following sequences of proper inclusions:

$A \subset S \subset W \subset I \subset R \subset P$ and $A_{0} \subset S_{0} \subset W_{0} \subset R_{0} \subset P_{0}$. Moreover, $B_{0} \subset B$, for each choice of $B=A, S, W, I, R, P$. (Note that $\theta \varepsilon A$ and $\left.\omega \varepsilon A_{o}.\right)$ These inclusions, as well as the counter-examples for properness, can be found amongst the results and examples of [4.5].

LEMMA 3.2. If $\rho_{g}$ is as in 2.3 , then $\rho_{g}$ belongs to $s$ (resp.A) if $\rho$ does.

Recall that if $\rho \varepsilon F$, then $\rho^{\prime} \varepsilon \mathrm{P}$; actually, more is true.

LEMMA 3.3. If $\rho \varepsilon F$, then $\rho^{\prime} \varepsilon \mathrm{S}$. Also, if $\rho \varepsilon \mathrm{S}$, then $\rho^{\prime \prime}=\rho$. PROOF. Let $\sigma=\|\cdot\|_{1}$. Then $\sigma \varepsilon S$ and hence, $\sigma_{g} \varepsilon S$, for all $g$ in $L^{\rho}$ such that $\rho(g) \leq 1(3.2)$. Moreover, $\rho^{\prime}=\sup \left\{\sigma_{g}: \rho(g) \leq 1\right\}$. Hence, $\rho^{\prime} \varepsilon S$, since $P$ and $S$ are closed under pointwise suprema (left to the reader). The second part of the lemma is well-known.

QUESTION 3.4. Recall that $A=\{\rho \varepsilon W: \rho$ is $A C N\}$. If $\rho \varepsilon P$ is $A C N$, is it automatically WFP, i.e., in W ?

4. SUPREMA.

We begin our study of the lattice theory of $P$ by asking if the subsets of $P$ introduced in Section 2 are closed under the formation of arbitrary pointwise 
suprema. We have already seen that $P$ and $S$ have this property (proof of 2.3). Our objective here is to summarize (for the sake of completeness) the known answers to this question for the other subsets.

4.1. $\mathrm{P}_{\mathrm{O}}$ is closed under the formation of arbitrary pointwise suprema. (In fact, if $\left\{\rho_{j}: j \in J\right\} \subseteq P$ and $\rho_{k}$ is a norm, for some $j=k$, then $\sup \left(\rho_{j}\right)$ is a norm.) The converse is false, i.e., it is possible for $\sup \left(\rho_{j}\right)$ to be a norm while all the $\rho_{j}$ are just semi-norms [4]. All that is required of the $\rho_{j}$ for their pointwise supremum to be a norm is that they be total, i.e., $\rho_{j}(f)=0$, all $\mathrm{j} \varepsilon \mathrm{J}$, implies $\mathrm{f}=0$.

4.2. $R_{0}$ and $I$ are closed under the formation of arbitrary pointwise suprema. This is not the case for $R[4, \mathrm{p} .147]$.

4.3 $\mathrm{W}$ and $\mathrm{W}_{\mathrm{O}}$ are closed under the formation of finite pointwise suprema. This is not true for infinite collections. In fact, there exists [4,p.150] a sequence in $W_{0}$ whose pointwise supremum is not in $W$.

Although $I_{0}=R_{0}$, we have seen that $W \subset I \subset R$. Furthermore, $I$ is closed under the formation of arbitrary pointwise suprema, while $W$ and $R$ are not. This is good reason for distinguishing the ITI property from WFP and RFP in general.

4.4. $S_{0}$ (as well as $S$ ) is closed under the formation of arbitrary pointwise suprema.

4.5. $A$ and $A_{0}$ are closed under the formation of finite pointwise suprema. This is not true for infinite collections. In fact, there exists a sequence in $A_{0}$ whose pointwise supremum is not in $A$.

- QUESTION 4.6. Is $R$ closed under the formation of finite pointwise suprema?

\section{INFIMA.}

In Section 1, we saw that the infimum in $P$ of two semi-norms $\rho_{1}, \rho_{2}$ is given by

$$
\left(\rho_{1} \wedge \rho_{2}\right)(\mathrm{f})=\inf \left\{\rho_{1}\left(\mathrm{f}_{1}\right)+\rho_{2}\left(\mathrm{f}_{2}\right): \mathrm{f}_{1}, \mathrm{f}_{2} \varepsilon \mathrm{M}^{+}, \mathrm{f}_{1}+\mathrm{f}_{2}=\mathrm{f}\right\}, \mathrm{f} \varepsilon \mathrm{M}^{+} \text {. }
$$

There are two cononical generalizations of this formula to the context of an arbitrary collection $\left\{\rho_{j}: j \varepsilon J\right\}$ of semi-norms. In this regard, it will be 
convenient to refer to a collection $\left(f_{j}\right)_{J}$ in $M^{+}$as a J-decomposition of $f$ in $\mathrm{M}^{+}$(of countable type) if :

(i) $\mathrm{f}_{j}=0$, for all but countably many $j$ in $\mathrm{J}$.

(ii) $\sum_{J} f_{j}=f$, in the almost-everywhere sense.

If $\left(f_{j}\right)_{J}$ has the property $\left(i^{\prime}\right) f_{j}=0$, for all but finitely many $j$ in $J$, then we will say it is of finite type. Now let $\mathrm{f} \varepsilon \mathrm{M}^{+}$and define:

$$
\lambda(f)=\inf \left\{\sum_{J} \rho_{j}\left(f_{j}\right):\left(f_{j}\right)_{J} \text { is a J-decomposition of } f\right\}
$$

and

$$
\gamma(f)=\inf \left\{\sum_{J} \rho_{j}\left(f_{j}\right):\left(f_{j}\right)_{J} \text { is a J-decomposition of } f \text { of finite type }\right\}
$$

Note that the sums in each case have at most countably many non-zero terms.

As suggested by Luxemburg in [3], there is another less canonical generalization of $\left(\rho_{1} \wedge \rho_{2}\right)(f)$ motivated by $\left(\rho_{1} \wedge \rho_{2}\right)^{\prime}=\rho_{1}^{\prime} \vee \rho_{2}^{\prime}$, namely,

$$
\sigma=\left(\sup \left(\rho_{j}^{\prime}\right)\right)^{\prime} \text {. }
$$

In this section, we shall make use of all three of these generalizations.

It is well-known that $\sigma$ is a semi-norm; in fact, $\sigma \varepsilon S$. Moreover:

THEOREM 5.1. Each of the mappings $\gamma, \lambda$ is a function semi-norm.

PROOF. The only part of the theorem for $\lambda$ needing verification is the monotonicity; we will show this. The proof for $\gamma$ is similar to that of $\lambda$. Suppose $f, g \in M^{+}$with $f \leq g$. Let $\left\{g_{k}\right\}$ be a sequence in $M^{+}$such that $\Sigma g_{k}=g$. Define $f_{1}=\min \left(f, g_{1}\right)$. Then $f_{1} \leq g_{1}$ and $f_{1} \leq f$, i.e., $\mathrm{f}_{1}^{\prime}=\mathrm{f}-\mathrm{f}_{1} \geq 0$. Suppose we have obtained $\mathrm{f}_{1}, \ldots, \mathrm{f}_{\mathrm{n}}$ in $\mathrm{M}^{+}$such that $f_{k} \leq g_{k}, 1 \leq k \leq n$, and $f_{1}+\ldots+f_{n} \leq f$, so that $f_{n}^{\prime}=f-f_{1}-\ldots-f_{n} \geq 0$. Define $f_{n+1}=\min \left(f_{n+1}^{\prime} g_{n+1}\right)$. Then $f_{n+1} \leq g_{n+1}$ and $\mathrm{f}_{\mathrm{n}+1} \leq \mathrm{f}_{\mathrm{n}}^{\prime}=\mathrm{f}-\mathrm{f}_{1}-\ldots-\mathrm{f}_{\mathrm{n}}$, i.e., $\mathrm{f}_{1}+\ldots+\mathrm{f}_{\mathrm{n}}+\mathrm{f}_{\mathrm{n}+1} \leq \mathrm{f}$. In this way, we obtain a sequence $\left\{f_{k}\right\}$ in $M^{+}$such that $f_{k} \leq f$ and $f_{k} \leq g_{k}, 1 \leq k$. We next show $\Sigma \mathrm{f}_{\mathrm{k}}=\mathrm{f}$ (in the almost-every-where sense).

We have $f(x) \leq g(x)$, for $x$ outside a null set. Let $x$ be a point in $x$ outside the null set. If $f(x)=g(x)$, then $f_{k}(x)=g_{k}(x)$, for all $k$, so 
that

$$
\Sigma \mathrm{f}_{\mathrm{k}}(\mathrm{x})=\Sigma \mathrm{g}_{\mathrm{k}}(\mathrm{x})=\mathrm{g}(\mathrm{x})=\mathrm{f}(\mathrm{x}) \text {. }
$$

If $f(x) \neq g(x)$, then $f(x)<g(x)$ and hence, $f_{i}(x) \neq g_{i}(x)$, for some $i$. Consequent1y,

$$
f_{i}(x)=f_{i-1}^{\prime}(x)=f(x)-f_{1}(x)-f_{1}(x)-\ldots-f_{i-1}(x),
$$

i.e.,

$$
f(x)=f_{1}(x)+\ldots+f_{i-1}(x)+f_{i}(x),
$$

which implies $\Sigma f_{k}(x)=f(x)$, i.e., $\Sigma f_{k}=f$.

Therefore, each decomposition of $g$ by a sequence $\left(g_{k}\right)$ in $M^{+}$yields a sequence decomposition $\left(f_{k}\right)$ of $f$ in $M^{+}$having the properties $f_{k} \leq g_{k}$, $1 \leq \mathrm{k}$. Consequent1y, the same is true for any $\mathrm{J}$-decomposition of $\mathrm{g}$ in $\mathrm{M}^{+}$, since such is essentially a sequence (define $f_{j}=0$, for $g_{j}=0$ ). Hence, if $\left(g_{j}\right)_{J}$ is a $J$-decomposition of $g$ in $\mathrm{M}^{+}$, there exists a corresponding J-decomposition $\left(f_{j}\right)_{J}$ of $f$ in $M^{+}$for which $f_{j} \leq g_{j}, j \varepsilon J$. Necessarily, $\lambda(f) \leq \lambda(g)$, since the infimum for $\lambda(f)$ is over a larger index set.

Observe that each $\lambda, \gamma, \sigma$ is a lower bound for $\left\{\rho_{j}: j \varepsilon J\right\}$.

Next we wish to compare $\gamma, \lambda, \sigma$. Obviously, $\lambda \leq \gamma$. Moreover, if $J$ is finite, then $\gamma=\lambda$.

LEMMA 5.2. If $\rho$ satisfies the ITI (i.e., $\rho \varepsilon I$ ) and $\rho$ is a lower bound for $\left\{\rho_{j}: j \in J\right\}$, then $\rho \leq \lambda$.

PROOF. Let $\mathrm{f} \varepsilon \mathrm{M}^{+}$and let $\left(\mathrm{f}_{\mathrm{j}}\right)_{\mathrm{J}}$ be a J-decomposition of $\mathrm{f}$ in $\mathrm{M}^{+}$. By our hypothesis, we have

from which follows that $\rho(f) \leq \lambda(f)$.

$$
\left.\rho(f)=\underset{J}{\rho} f_{j}\right) \leq \sum_{J} \rho\left(f_{j}\right) \leq \sum_{J} \rho_{j}\left(f_{j}\right),
$$

THEOREM 5.3. Let $\left\{\rho_{j}: j \varepsilon J\right\}$ be a subset of $P$ with $r, \lambda, \sigma$ as above. Then $\sigma<\lambda<\gamma$ and $\sigma=\gamma^{\prime \prime}=\lambda^{\prime \prime}=\sigma^{\prime \prime}$.

PROOF. Since $\sigma \varepsilon S \subset I$ and $\sigma \leq \rho_{j}, j \varepsilon J$, we have $\sigma \leq \lambda \leq Y$ (5.2). Hence, $\sigma=\sigma^{\prime \prime} \leq \lambda^{\prime \prime} \leq \gamma^{\prime \prime}$. Now $\gamma \leq \rho_{j}$ implies $\gamma^{\prime} \geq \rho_{j}^{\prime}, j \varepsilon \mathrm{J}$, i.e., $\gamma^{\prime}$ is an upper bound for $\left\{\rho_{j}^{\prime}: j \varepsilon J\right\}$. Thus, $\gamma^{\prime} \geq \sigma^{\prime}$, since $\sigma^{\prime}=\sup \left(\rho_{j}^{\prime}\right)$. Consequently, $\gamma^{\prime \prime} \leq \sigma^{\prime \prime}=\sigma$ and $\sigma=\gamma^{\prime \prime}$. This establishes the equalities. The 
following examples establish the inequalities.

EXAMPLE 5.4. Let $X=\mathbb{N}$. Define

$$
\alpha(f)=\Sigma f(n) / 2^{n}, \beta(f)=1 \text { im } \sup f(n)
$$

and

$$
\rho_{j}(f)=\alpha(f)+\beta(f), f \varepsilon M^{+}, j=1,2, \ldots,
$$

so that $\left\{\rho_{j}\right\}$ is a constant sequence of norms. We leave to the reader the task of verifying that $\lambda<\gamma$ for this choice of $\left\{\rho_{j}\right\}$.

EXAMPLE 5.5. Let $\rho$ be a non-trivial element of $P$ such that $\rho \notin S$, i.e., $\rho^{\prime \prime} \neq \rho$, in which case $\rho^{\prime \prime}<\rho$. For the collection $\left\{\rho_{j}\right\}=\{\rho, 2 \rho\}$, we have $\sigma=\rho^{\prime \prime}<\rho=\lambda$, i.e., $\sigma<\lambda$.

LEMMA 5.6. For finite collections in $\mathrm{S}$, we have $\sigma=\lambda=\gamma$ (use 2.8). For the remainder of this section, we investigate the status of the infimum in $P, R, I, W, S, A$. Once again, for $\left\{\rho_{j}: j \varepsilon J\right\} \subseteq P$, let $\sigma, \lambda, \gamma$ be as above.

LEMMA 5.7. The infimum of the $\rho_{j}$ in $P$ is $r$; notationally, $\gamma=\inf _{p}\left(\rho_{j}\right)$. In particular, $\rho_{1} \wedge \rho_{2}=\inf _{p}\left(\rho_{1}, \rho_{2}\right)$. In this sense, $P$ is closed under the formation of arbitrary infima.

LEMMA 5.8. The infimum of a family in $P_{0}$ which is bounded below in $P_{0}$ also belongs to $P_{0}$. This is not true for arbitrary families in $P_{0}$. (For example, consider $\{(1 / n) \rho\}$, for $\left.\rho \in P_{0} \cdot\right)$

THEOREM 5.9. If $\left\{\rho_{j}\right\} \subseteq I$, then the infimum of the $\rho_{j}$ in $I$ is $\lambda$; notationally, $\inf _{I}\left(\rho_{j}\right)=\lambda$. In particular, $\rho_{1} \wedge \rho_{2}=\inf _{I}\left(\rho_{1}, \rho_{2}\right)$. In this sense, I is closed under the formation of arbitrary infima.

PROOF. Fix $\varepsilon>0$ and let $\left\{g_{n}\right\}$ be a sequence in $M^{+}$for which $\Sigma \lambda\left(g_{n}\right)<\infty$. By the definition of $\lambda$, for each $n$, there exists a $J^{n}$-decomposition $\left(g_{n, j}\right)^{n}$ of $g_{n}$ in $M^{+}$such that

$$
\sum_{J^{n}} \rho_{j}\left(g_{n, j}\right)<\lambda\left(g_{n}\right)+\varepsilon / 2^{n}
$$

Let $J_{n}=\left\{j \in J^{n}: g_{n, j} \neq 0\right\}$. Then $J_{n}$ is a countable subset of $J^{n}$ and

$$
\sum_{J^{n}} \rho_{j}\left(g_{n, j}\right)=\sum_{J_{n}} \rho_{j}\left(g_{n, j}\right), n=1,2, \ldots
$$


Let $J=U J_{n}$, which is also a countable subset of $U J^{n}$. Furthermore, if $j \notin J$, then $g_{n, j}=0$, all $n$. Observe that

$$
\begin{aligned}
\sum_{n J_{n}} \rho_{j}\left(g_{n, j}\right) & <\sum_{n}\left[\lambda\left(g_{n}\right)+\varepsilon / 2^{n}\right] \\
& \leq \underset{n}{\sum} \lambda\left(g_{n}\right)+\varepsilon \\
& <\infty .
\end{aligned}
$$

Therefore, rearranging the double series, we obtain

$$
\begin{aligned}
\sum_{n J_{n}} \rho_{j}\left(g_{n, j}\right) & =\sum_{n} \sum_{J} \rho_{j}\left(g_{n, j}\right) \\
& =\sum_{J} \sum_{n} \rho_{j}\left(g_{n, j}\right),
\end{aligned}
$$

Moreover, $\left\{g_{n, j}: j \in J, n \varepsilon \mathbb{N}\right\}$ is a countable subset of $M^{+}$satisfying

$$
\begin{array}{lll}
\sum \sum & g_{n, j}=\sum g_{n} \\
n & J &
\end{array}
$$

Thus, $\left\{g_{n, j}\right\}$ is a subset of $M^{+}$which is indexed by $\mathbb{N} \times J$, has countably many elements and satisfies

$$
\sum_{J \text { n }}^{\sum \sum} g_{n, j}=\sum_{n} \sum_{J} g_{n, j}=\sum_{n} g_{n}
$$

Thus, the family $\underset{n}{\left(\sum g_{n, j}\right)_{J}}$ is a J-decomposition of $\sum_{n} g_{n}$ is $M^{+}$. Consequent1y,

$$
\begin{aligned}
\underset{n}{\lambda\left(g_{n}\right)} & \leq \sum_{J} \rho_{j}\left(\sum_{n} g_{n, j}\right) \\
& \leq \sum_{J} \sum_{n} \rho_{j}\left(g_{n, j}\right) \quad\left(\rho_{j} \varepsilon I\right) \\
& \leq \sum_{n} \sum_{n} \rho_{j}\left(g_{n, j}\right) \\
& <\underset{n}{\sum} \lambda\left(g_{n}\right)+\varepsilon, \text { for arbitrary } \varepsilon>0 .
\end{aligned}
$$


Hence,

$$
\lambda\left(\sum_{n} g_{n}\right) \leq \underset{n}{\Sigma} \lambda\left(g_{n}\right)
$$

which implies that $\lambda \varepsilon I$.

If $\rho$ is in $I$ and $\rho$ is a lower bound for $\left\{\rho_{j}\right\}$, then by $5.2 \rho \leq \lambda$. Hence, $\lambda=\inf _{I}\left(\rho_{j}\right)$.

COROLLARY 5.10. If $\left\{\rho_{j}\right\} \subseteq R_{0}=I_{0}$ and $\lambda$ is a norm, then $\lambda$ is the infimum of the $\rho_{j}$ if $R_{o}$.

COROLLARY 5.11. If $\rho_{1}, \rho_{2} \varepsilon R_{0}$ and $\rho_{1} \wedge \rho_{2}$ is a norm, then $\rho_{1} \wedge \rho_{2}$ is the infimum of $\rho_{1}, \rho_{2}$ in $R_{0}$.

PROPOSITION 5.12. If $\rho_{1}, \rho_{2} \varepsilon W$, then $\rho_{1} \wedge \rho_{2}$ is the infimum of $\rho_{1}, \rho_{2}$ is $W$. Thus, $W$ is closed under the formation of finite infima.

PROOF. Recall that $\tau$ belongs to $W$ if and only if $\tau$ is equivalent to $\tau^{\prime \prime}[5, \mathrm{p} .472]$, i.e., there exists $0<\mathrm{a} \leq 1$ such that $a \tau \leq \tau " \leq \tau$. Thus, for $\rho_{1}, \rho_{2} \varepsilon W$, there exists $0<a_{i} \leq 1$ such that $a_{i} \rho_{i} \leq \rho_{i} " \leq \rho_{i}$, $i=1,2$. Letting $a=\min \left(a_{1}, a_{2}\right)$, we have $0<a \leq 1$ and $a \rho_{i} \leq \rho_{i} " \leq \rho_{i}$, $i=1,2$. From this it follows that

$$
a\left(\rho_{1} \wedge \rho_{2}\right) \leq \rho_{1} " \wedge \rho_{2}^{\prime \prime} \leq \rho_{1} \wedge \rho_{2},
$$

i.e., $\rho_{1} \wedge \rho_{2}$ is equivalent to $\rho_{1} " \wedge \rho_{2} "$. However, by 2.8 we have that $\rho_{1} " \wedge \rho_{2} "=\left(\rho_{1} \wedge \rho_{2}\right) "$. Hence, $\rho_{1} \wedge \rho_{2} \varepsilon \mathrm{W}$.

COROLLARY 5.13. If $\rho_{1}, \rho_{2} \varepsilon \mathrm{W}_{\mathrm{o}}$ and $\rho_{1} \wedge \rho_{2} \varepsilon \mathrm{P}_{\mathrm{o}}$, then $\rho_{1} \wedge \rho_{2} \varepsilon \mathrm{W}_{\mathrm{o}}$.

PROPOSITION 5.14. If $\left\{\rho_{j}\right\} \subseteq S$, then the infimum of the $\rho_{j}$ in $s$ is $\sigma$; notationally, $\sigma=\inf _{S}\left(\rho_{j}\right)$. In particular, $\rho_{1} \wedge \rho_{2}=\inf _{S}\left(\rho_{1}, \rho_{2}\right)$ (recall 5.6). In this sense, $S$ is closed under the formation of arbitrary infima.

PROOF. We have seen that $\sigma$ is a lower bound for $\left\{\rho_{j}\right\}$. If $\rho$ in $S$ is any lower bound for $\left\{\rho_{j}\right\}$, then $\rho^{\prime} \geq \rho_{j}^{\prime}, j \varepsilon J$, so that $\rho^{\prime} \geq \sup \left(\rho_{j}^{\prime}\right)$. Consequently, $\rho^{\prime \prime} \leq\left(\sup \left(\rho_{j}^{\prime}\right)\right)^{\prime}$, i.e., $\rho \leq \sigma$.

PROPOSITION 5.15. The infimum of a family in $S_{o}$ which is bounded below $S_{0}$ is also in $P_{0}$. This is not true for arbitrary families in $S_{0}$ (5.8). 
PROOF. By 5.14, $\sigma=\inf _{S}\left(\rho_{j}\right)$ belongs to $s$, for $\left\{\rho_{j}\right\} \subseteq S_{o}$ and bounded below in $S_{0}$. In order that $\sigma \varepsilon S_{0}$, it is necessary and sufficient that $\sup \left(\rho_{j}^{\prime}\right)$ be saturated [5,p.458]. By hypothesis, there exists $\rho$ in $s_{0}$ such that $\rho \leq \rho_{j}, j \varepsilon J$. Hence, $\rho^{\prime} \geq \rho_{j}^{\prime}, j \varepsilon J$, so that $\rho^{\prime} \geq \sup \left(\rho_{j}^{\prime}\right)$, where $\rho^{\prime}$ is saturated $[5, p .458]$. Therefore, $\sup \left(\rho_{j}^{\prime}\right)$ is also saturated $[5$, p.454], i.e., $\sigma$ is a norm.

PROPOSITION 5.16. If $\rho_{1}, \rho_{2} \varepsilon A$, then $\rho_{1} \wedge \rho_{2}$ is the infimum of $\rho_{1}, \rho_{2}$ in A. Thus, A is closed under the formation of finite infima.

QUESTION 5.17. Are $P_{0}, R$ closed under the formation of finite infima?

\section{SUBLATTICES AND COMPLETENESS}

We are now prepared to determine (as well as we can) which of the subsets introduced in Section 3 are sublattices of $P$. For those which are, we are a1so interested in whether or not they are complete. This section is essentially a lattice-theoretic summary of the previous three sections.

With regard to completeness, it is worthwhile recalling the following. A lattice is complete if it is closed under arbitrary suprema and infima. A lattice is Dedekind complete if it is closed under arbitrary suprema and infima of collections which are suitably bounded above for suprema and below for infima. Since $\theta \varepsilon A$ and $\omega \varepsilon A_{0}$, these two notions of completeness are usually the same - but not always.

As a consequence of the results of Section 5, we see that it is possible for a subset of a sublattice to have an infimum in the sublattice which is different from its infimum in the whole lattice. Therefore, it is possible for a sublattice to be a complete lattice, but not a complete sublattice!

THEOREM 6.1. $\mathrm{P}$ is a complete lattice with supremum given pointwise and infimum given by $Y$ of Section 5 . (See 2.5,2.6,4.1, and 5.7.)

REMARKS 6.2. It is not clear whether $P_{0}$ is a sublattice of $P$, since we do not know if $P_{0}$ is closed under finite infima. For this reason, it is also difficult to determine the sublattice status of $R_{0}, W_{0}, S_{0}, A_{0}$. However, $P_{0}$ is Dedekind complete in the sense that: (i) it is closed under the formation of 
arbitrary pointwise infima and (ii) it is closed under the formation of $\mathrm{r}$-infima of arbitrary collections bounded below in $\mathrm{P}_{0}$.

THEOREM 6.3. I is a sublattice of $P$ with supremum given pointwise and infimum given by $\lambda$ of Section 5 . Thus, $I$ is also a complete lattice (4.2, 5.9), but not a complete sublattice of $P$ (next example).

EXAMPLE 6.4. Let $X=\mathbf{N}$ and define

$$
\rho_{j}(f)=\operatorname{supf}_{n \geq j}(n), f \varepsilon M^{+}, j=1,2, \ldots,
$$

so that $\rho_{j} \varepsilon S$, all $j$. For the collection $\left\{\rho_{j}: j \varepsilon \mathbb{N}\right\}$, the corresponding $\gamma$ is given by

$$
r(f)=\lim \sup (f(n)), f \varepsilon M^{+}
$$

We may verify that $\Upsilon \notin I$, i.e., inf ${ }_{p}\left(\rho_{j}\right) \notin I$.

THEOREM 6.5. $W$ is a sublattice of $I$ which is not (Dedekind) complete in general $(4.3,5.12)$.

THEOREM 6.6. $\mathrm{S}$ is a sublattice of $\mathrm{W}$ with supremum given pointwise and infimum given by $\sigma$ of Section 5. Thus, $\mathrm{S}$ is also a complete lattice, but not a complete sublattice of $\mathrm{W}$.

PROOF. See 4.4 and 5.15. That $S$ is not complete in $W$ can be seen from the example in 7.3. For this choice of $\left\{\rho_{n}\right\}$, we may verify that

$$
\lambda(f)=\gamma(f)=\sup (f(n))+1 i m \sup (f(n)), f \varepsilon M^{+} \text {. }
$$

Then $\rho_{\mathrm{n}} \varepsilon \mathrm{S}_{\mathrm{o}}, \mathrm{all} \mathrm{n}, \lambda \varepsilon \mathrm{W}[4, \mathrm{p} .150], \lambda \notin \mathrm{s}$, while $\sigma \varepsilon \mathrm{S}$. Thus we have another example of $\sigma \neq \lambda$ (recall 5.5).

THEOREM 6.7. A is a sublattice of $\mathrm{S}$ which is not (Dedekind) complete. PROOF. See 4.6 and 5.16 .

\section{DEMORGAN'S LAWS}

In view of 2.8 , it is natural to ask if DeMorgan's Laws hold in general. Once again, let $\left\{\rho_{j}: j \in J\right\} \subseteq P$. The next theorem shows in what sense one of the two DeMorgan Laws is valid.

THEOREM 7.1. In general, $\left[\inf { }_{P}\left(\rho_{j}\right)\right]^{\prime}=\sup \left(\rho_{j}^{\prime}\right) \cdot$ 
PROOF. For convenience, let $\gamma=\inf { }_{P}\left(\rho_{j}\right)$ and $\beta=\sup \left(\rho_{j}^{\prime}\right)$. We then have

$$
\gamma^{\prime}(f)=\sup \left\{\int_{X}|f g| d \mu: Y(g) \leq 1\right\}, f \in M^{+} \text {. }
$$

To show $\gamma^{\prime}=\beta$, first observe that $\gamma^{\prime} \geq \beta$ since $\gamma \leq \rho_{j}$, i.e., $\gamma^{\prime} \geq \rho_{j}^{\prime}, j \varepsilon \mathrm{J}$. Suppose next that $\mathrm{f} \varepsilon \mathrm{M}^{+}, \gamma(g) \leq 1$ and $\left(g_{j}\right)$ is a J-decomposition of $|\mathrm{g}|$ in $\mathrm{M}^{+}$of finite type. Since $\gamma(g) \leq 1$, we may assume $\sum \rho_{j}\left(g_{j}\right)<\infty$, i.e., $\rho_{j}\left(g_{j}\right)<\infty, j \varepsilon J$. If $\beta(f)=\infty$, then $\gamma^{\prime}(f)=\infty$ also. If $\beta(f)<\infty$, then $\rho_{j}^{\prime}(f)<\infty, j \varepsilon J$, and hence

$$
\begin{aligned}
\int_{X}|f g| d \mu & =\sum \int_{X} f g_{j} d \mu \quad \text { (finite sum) } \\
& \leq \sum_{J} \rho_{j}^{\prime}(f) \quad \rho_{j}\left(g_{j}\right) \quad \text { (Hölder's Inequality) } \\
& \leq \beta(f) \quad \sum_{J} \rho_{j}\left(g_{j}\right) .
\end{aligned}
$$

Thus, from the definition of $\gamma$, it follows that

$$
\begin{aligned}
\int_{X}|f g| d \mu & \leq \beta(f) \gamma(g) \\
& \leq \beta(f) .
\end{aligned}
$$

Thus,

$$
\gamma^{\prime}(f) \leq \beta(f), \text { i.e., } \gamma^{\prime} \leq \beta \text {. }
$$

This theorem yields an alternate proof of the equality in 5.3. Specifically, we have:

COROLLARY 7.2. Let the notation be as in 5.3. Then $\sigma=\gamma^{\prime \prime}$.

PROOF. Since $\gamma=\inf _{\mathrm{P}}\left(\rho_{j}\right)$, we have $\gamma^{\prime}=\sup \left(\rho_{j}^{\prime}\right)$ by the theorem, so that $\gamma^{\prime \prime}=\left(\sup \left(\rho_{j}^{\prime}\right)\right)^{\prime}=\sigma$.

In general, as the next example verifies, the other DeMorgan Law is false. EXAMPLE 7.3. Let $\mathrm{X}=\mathbb{N}$. Define

$$
\rho_{n}(f)=\sup _{k}(f(k))+\sup _{k \geq n}(f(k)), \text { f } \varepsilon M^{+}, n=1,2, \ldots
$$


We may verify that:

(i) each $\rho_{\mathrm{n}}$ is an SFP norm, i.e., $\rho_{\mathrm{n}} \varepsilon \mathrm{S}_{\mathrm{o}}, \mathrm{n}=1,2, \ldots$,

(ii) $\inf _{\mathrm{P}}\left(\rho_{\mathrm{n}}\right)(\mathrm{f})=\sup _{\mathrm{k}}(\mathrm{f}(\mathrm{k}))+\underset{k}{\lim \sup (f(k))}$, f $\varepsilon \mathrm{M}^{+}$.

(iii) in $f_{P}\left(\rho_{n}\right)$ does not have the SFP.

Consequently, letting $\sigma_{n}=\rho_{n}{ }^{\prime}$, we see that $\inf { }_{p}\left(\sigma_{n}{ }^{\prime}\right)=\inf f_{P}\left(\rho_{n}\right)$ does not have the SFP while $\left(\sup \left(\sigma_{n}\right)\right)^{\prime}$ does. Therefore, $\left(\sup \left(\sigma_{n}\right)\right)^{\prime}$ can't equal $\inf { }_{\mathrm{P}}\left(\sigma_{\mathrm{n}}{ }^{\prime}\right)$.

Despite the previous example, there is a partial result of interest.

LEMMA 7.4. If $\left\{\rho_{j}: j \varepsilon J\right\} \subseteq P$, then $\left(\sup \left(\rho_{j}\right)\right)^{\prime} \leq \inf { }_{S}\left(\rho_{j}^{\prime}\right)(\operatorname{apply} 2.7)$. However, $\quad \inf \mathrm{S}_{\mathrm{S}}\left(\rho_{\mathrm{j}}\right)^{\prime}=\sup \left(\rho_{\mathrm{j}}^{\prime}\right)$.

of course, for finite families $\left\{\rho_{j}\right\}$, it is true that $\left(\sup \left(\rho_{j}\right)\right)^{\prime}=\inf f_{p}\left(\rho_{j}\right)$ $\inf _{S}\left(\rho_{j}\right)($ recall 2.8).

8. CONCLUDING REMARKS.

A lattice-theoretic question which we have not yet considered is whether $P$ (or any of its sublattices) is distributive. As indicated by Luxemburg in [3], the answer is "no" in general. More specifically, there exist norms $\rho_{1}, \rho_{2}, \rho_{3}$ in $A_{0}$ for which

$$
\left(\rho_{1} \vee \rho_{2}\right) \wedge \rho_{3}>\left(\rho_{1} \wedge \rho_{3}\right) \vee\left(\rho_{2} \wedge \rho_{3}\right)
$$

thus violating one of the distributive laws. As a consequence of this, the other distributive law is also violated, in general, because

$$
\left(\rho_{1}^{\prime} \wedge \rho_{2}^{\prime}\right) \vee \rho_{3}^{\prime}<\left(\rho_{1}^{\prime} \vee \rho_{3}^{\prime}\right) \wedge\left(\rho_{2}^{\prime} \vee \rho_{3}^{\prime}\right)
$$

Therefore, both distributive laws fail in $A_{0}$ and hence, in $P$ and each of its sublattices of interest.

Finally, observe that at first glance, finite sums seem to have nothing to do with lattice theory of function semi-norms. However, quite the opposite is true. If $\left\{\rho_{1}, \ldots, \rho_{n}\right\} \subset P$, then $\rho_{1}+\ldots+\rho_{n} \varepsilon P$ and is equivalent to $\sup \left(\rho_{1}, \ldots, \rho_{n}\right)$. Consequently, its associate $\left(\rho_{1}+\ldots+\rho_{n}\right)^{\prime}$ is equivalent to in $\tilde{i}_{\mathrm{P}}\left(\rho_{1}^{\prime}, \ldots, \rho_{\mathrm{n}}^{\prime}\right)$.

In view of the previous, it is of interest to know which of the subsets of 
$\mathrm{P}$ (introduced in Section 2) are closed under equivalence. The spaces $\mathrm{P}_{\mathrm{o}}, \mathrm{R}, \mathrm{I}, \mathrm{W}$ are closed under equivalence; $\mathrm{S}$ isn't. Is $\mathrm{A}$ ?

ACKNOWLEDGEMENT. Both authors were partially supported by Oakland University Research Fellowships.

The authors are grateful to W.A.J. Luxemburg for providing them with a copy of [3], as well as for a very helpful personal communication.

\section{REFERENCES}

1. Halmos, P.R., Measure Theory, Van Nostrand, Princeton, 1962.

2. Köthe, G., Topological Vector Spaces I, Springer-Verlag, New York, 1969.

3. Luxemburg, W.A.J., Sup and inf constructions for saturated function norms, unpublished preprint.

4. Luxemburg, W.A.J., and Zaanen, A.C., Notes on Banach function spaces I, II, Indag. Math. 25(1963), 135-153.

5. Zaanen, A.C., Integration, North Hollard, Amsterdam, 1967. 


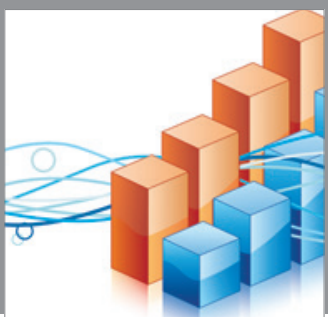

Advances in

Operations Research

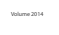

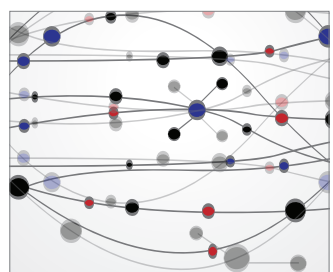

\section{The Scientific} World Journal
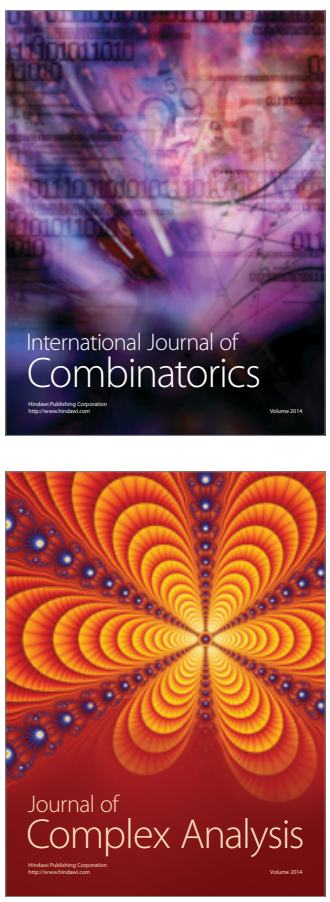

International Journal of

Mathematics and

Mathematical

Sciences
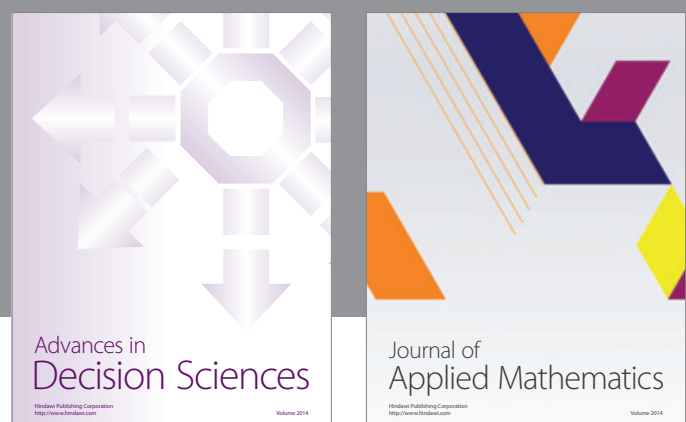

Journal of

Applied Mathematics
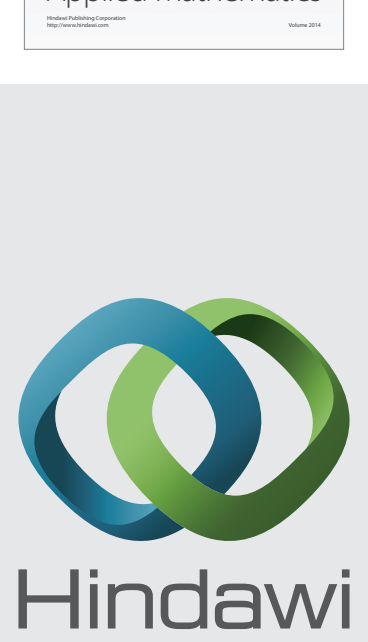

Submit your manuscripts at http://www.hindawi.com
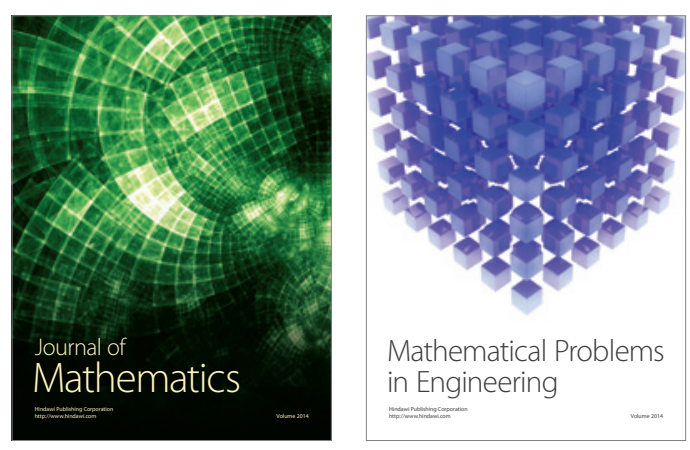

Mathematical Problems in Engineering
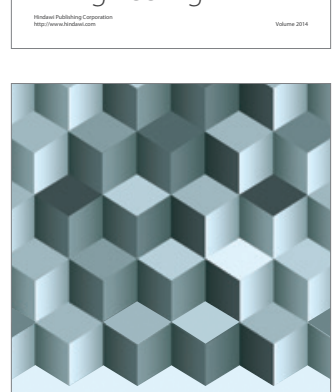

Journal of

Function Spaces
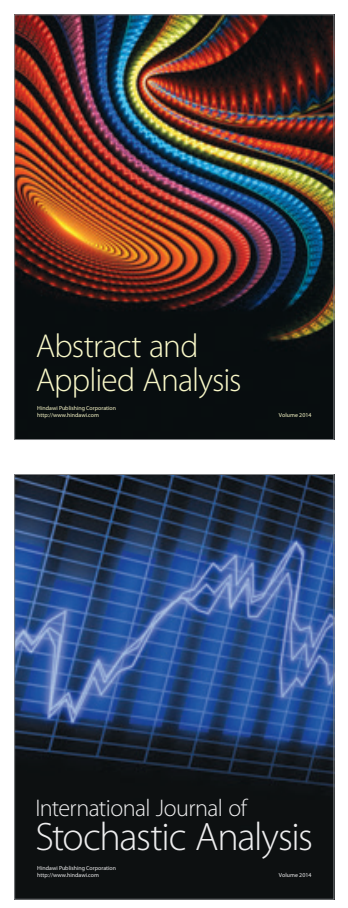

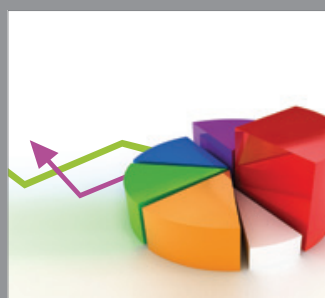

ournal of

Probability and Statistics

Promensencen
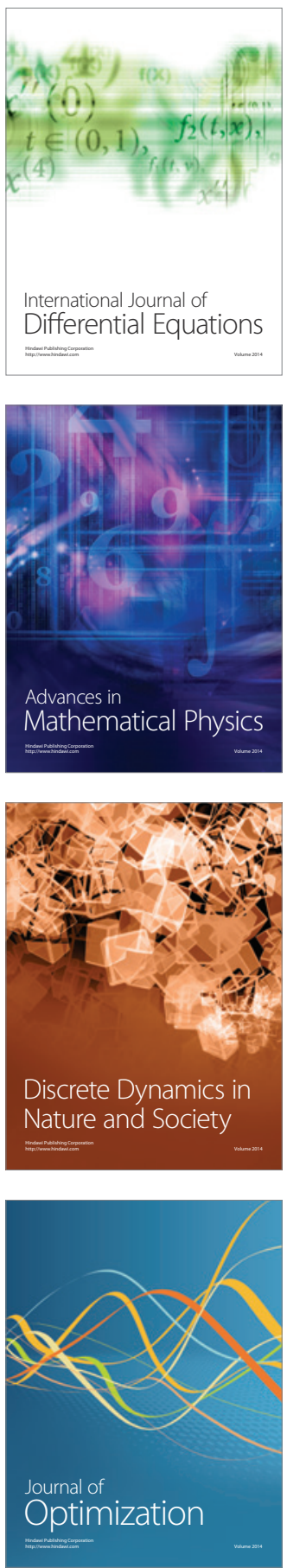